\title{
EL CONTRACHAPAdO ESTRUCTURAL ${ }^{(1)}$. Vicente Pérez
}

Galaz, Ingeniero Civil, $U$. de Chile, Profesor Asociado, Departamento de Ingeniería en Obras Civiles, Facultad de Ingeniería, Universidad de Santiago de Chile, Avda. B. O'Higgins 3363, Santiago-Chile.

\section{RESUMEN}

El presente articulo entrega antecedentes necesarios para entender de una mejor forma lo que significa la expresión "contrachapado estructural". Ella involucra tener presente la calidad de las chapas de las caras exteriores del tablero, el tipo de adhesivo usado entre chapas y la calidad estructural del tablero resultante, la cual determina las tensiones admisibles que se deben usar en el cálculo de elementos estructurales que incluyan a este material entre sus componentes. Se han considerado como antecedentes las normas australianas $y$ norteamericanas que existen, actualmente, sobre la materia.

El trabajo está orientado a la ilustración de usuarios, promotores del material y de algunos productores que, a veces, usan el término "contrachapado estructural" como una marca comercial, olvidando determinar y entregar los valores numéricos de sus propiedades resistentes y geométricas, sin los cuales no es posible dimensionar el elemento estructural para las cargas a las cuales estará sometido.

CONICYT, a través del financiamiento de sus Proyectos FONDECYT, hace posible esta investigación, la que tiene como objetivo conocer algunas propiedades teóricas y experimentales del contrachapado que se fabrica en el pais.

Palabras Clave : Madera, Contrachapado, Estructuras.

\section{ABSTRACT}

A general background for a better understanding of " structural plywood " concept is presented. The term "structural plywood" is related to a given finishing quality of the exterior faces of the board, the type of adhesive utilized to join the multiple veneers and the resulting quality of the plywood sheets.

The objective of this work is to describe the main mechanical properties of the structural plywood. Structural plywood quality determines its mechanical properties, that is the workings loads that should be used in the design process of structural elements containing structural plywood. Australian and American codes have been considered as references.

This work is aimed at to illustrate users, promotors and some manufacturers, who sometimes simply employ the term "structural plywood" as a trade mark no mentioning the working stress values at all. Without mechanical properties information on the product, structural design is not possible.

The author is grateful to CONICYT (National Comission of Scientific and Technological Research) who through FONDECYT research proyects funding made this work possible.

Keywords : Timber, Plywood, Structures. 


\section{INTRODUCCIÓN}

El contrachapado es un producto de la madera, en forma de tablero, conformado por chapas de madera encoladas entre sí de modo que la dirección de la fibra de algunas tulipas quede perpendicular al eje longitudinal del tablero y otras tengan dirección paralela a dicho eje. En la mayoria de los tipos de contrachapado, la dirección de las fibras de la caras exteriores se deja paralela al eje longitudinal del tablero. Por lo tanto, para mantener éste balanceado, se usa un número impar de chapas, alternándose la dirección de las fibras.

Sin embargo, algunos tableros de contrachapado se producen con un número par de chapas, en tal caso se deben dejar dos chapas con la fibra en la misma dirección para conformar la parte central del tablero.

El contrachapado también se construye usando madera cepillada o tableros de partículas en el centro, recubriéndolos con una chapa de madera en ambas caras. Estos tipos de tableros se usan con frecuencia en la industria del mucble y en algunos casos en la construcción.

A pesar que la industria del contrachapado sólo data del año 1905 , la chapa tiene una historia mucho más extensa. Se sabe que los egipcios ( 1500 A.C.) fabricaban chapas para decorar muebles. Los griegos y romanos también ocuparon métodos para cortar chapas. El gran avance de la Industria del Contrachapado se produce en 1930. La adopción de la prensa caliente y cl desarrollo de los adhesivos a base de resinas sintéticas se reconocen como los hitos principales para su impulso definitivo.

La mayor parte de la tecnologia para la industria del contrachapado confeccionado con coniferas se desarrolló en Estados Unidos. La Segunda Guerra Mundial aceleró tal tecnología para fabricar tableros contrachapados para ser usados en exteriores, mediante el empleo de adhesivos derivados de resinas fenólicas, los cuales se utilizaron para la fabricación de naves pequeñas de combate. En la década del 70 otras áreas adoptaron esta técnica para producir contrachapado a base de trozas de pequeño diámetro.

La tecnología para producir contrachapado con especies latifoliadas difiere considerablemente de aquella usada para producir este material con especies coníferas. Los grados de calidad y sus usos difieren notablemente para latifoliadas y coníferas. Los contrachapados de coniferas se usan. principalmente. en la construcción. Para éstos se emplean chapas gruesas y bastas y se fabrican tanto con caras sin lijado. para revestimientos y pisos que posteriormente serán recubiertos. como cepilladas para una gran variedad de usos tanto decorativos como estructurales.

Las chapas producidas con especies latifoliadas son usadas para fabricar paneles decorativos. clementos aislados para la industria. mucbles y tambićn como material de 
construcción. La industria del contrachapado de latifoliadas es muy diversa en los países desarrollados: Comprende desde elementos solicitados por el consumidor para aplicaciones arquitectónicas específicas, hasta empresas con líneas de producción mayor, similares a las mencionadas para el tablero contrachapado de coníferas.

El contrachapado se ha convertido en un valioso material, debido a que tiene propiedades únicas e ideales para la construcción : Es liviano y sin embargo resistente, es un tablero por lo que puede cubrir grandes áreas de pisos, muros o techumbres manipulando sólo unas pocas piezas. Puede ser clavado, encolado y cortado con la misma facilidad que la madera. Su primer gran uso, allá por 1910 , fue la fabricación de puertas. Desde entonces, este material ha reemplazado a la madera en muchas aplicaciones, de las cuales se destaca el revestimiento de muros, pisos y techumbres. En las últimas décadas el contrachapado fabricado con coniferas ha permitido el progreso de la industria del prefabricado de elementos destinados a la industria de la vivienda.

El contrachapado tiene algunas ventajas sobre la madera, pero no es más resistente en todos los aspectos. Una franja de contrachapado entre dos apoyos no soporta más carga que un trozo de madera de las mismas dimensiones. En tal situación la madera será un poco más resistente. Sin embargo, el contrachapado proporciona resistencia a la flexión en ambas direcciones de su plano, por lo que es muy conveniente como cubierta de pisos. de techumbre y de muros, esté paralelo o normal a las vigas que lo sostienen. Otra ventaja del contrachapado es su forma rectangular rígida, lo que hace casi imposible deformarlo mediante una fuerza con dirección paralela al plano del tablero.

Esta propiedad explica el buen comportamiento de muros exteriores, pisos y techumbres en viviendas o estructuras sometidas a fuerzas sísmicas o de vientos de alta velocidad.

En Chile es cada vez mayor la utilización del contrachapado derivado de especies latifoliadas. En los últimos años se ha estado fabricando contrachapado de pino radiata, para la construcción. Sin embargo su uso ha estado limitado por el desconocimiento que los industriales y profesionales de la construcción tienen del nombre " contrachapado estructural " y de lo que en realidad involucra.

\section{DEFINICIÓN DEL CONTRACHAPADO ESTRUCTURAL}

Se entiende por " contrachapado estructural " a un tablero con propiedades físicas y mecánicas o estructurales definidas ( conocidas ).

Las propiedades fisicas que se deben conocer son :

Contenido de Humedad media del tablero. densidad o peso específico, contracciones y expansiones. peso por unidad de superficie y dimensiones finales (espesor, ancho y longitud). 
Las propiedades mecánicas que se deben determinar son:

Tensiones de flexión en el límite de proporcionalidad, módulo de ruptura y módulo de elasticidad para carga aplicada en la cara del tablero, con direcciones de la fibra de las caras exteriores paralela y normal a la luz (distancia entre apoyos) de la pieza.

Tensiones de flexión en el límite de proporcionalidad, módulo de ruptura y módulo de elasticidad, para carga aplicada en el canto de la pieza con direcciones de la fibra de las caras exteriores paralela y normal a la luz.

Tensiones máximas o de ruptura a la compresión, para carga aplicada en el canto de la pieza, con direcciones de la fibra de las caras exteriores paralela y normal a la dirección de la carga.

Tensiones máximas o de ruptura a la tracción. para carga aplicada en el canto de la pieza, con direcciones de la fibra de las caras exteriores paralela y normal a la dirección de la carga.

Tensiones máximas o de ruptura al cizalle a través del espesor. para carga aplicada en el canto de la pieza. con direcciones de la fibra de las caras exteriores paralela y normal a la dirección de la carga.

Tensiones máximas o de ruptura de cizalle entre chapas, para cargas aplicadas en las caras de las piezas con dirección de la fibra de las caras exteriores normal a la dirección de la carga.

Módulo de cizalle en el plano del tablero (G).

Si de un tablero se desconocen estas propiedades mecanicas, con el no es posible el calculo de elementos estructurales y por lo tanto a dicho tablero no se le puede definir como estructural.

En los diferentes paises desarrollados se han establecido normas para la determinación de éstas propiedades de los tableros. Las más conocidas y usadas son las Normas de la American Society for Testing and Matcrials (ASTM). También se destacan las normas de la American Plywood Association (APA) y las normas australianas Australian Standards (AS).

En el presente trabajo se revisarán las Normas AS, pues son las más simples de aplicar en un pais como Chile. con pocas especies forestales. que está iniciando su normalización en este campo. Además, los criterios de normalización australianos ya fueron incluidos en las Normas Chilenas. especificamente para determinar las Tensiones Admisibles de las maderas comerciales crecidas en el pais ( Ver NCh 1198 ). 


\section{COMPARACIÓN ENTRE CONTRACHAPADO Y MADERA}

El contrachapado puede estar compuesto de chapas de diferentes espesores, de distintas especies madereras y de diversas calidades estructurales. Las chapas, en el contrachapado, tienen una leve diferencia de propiedades estructurales con las correspondientes a las de la madera origen. Las causas de ello son:

En el momento de la manufactura las chapas tienen numerosas grietas, las cuales son parcialmente rellenadas por el adhesivo durante el prensado.

El bajo contenido de humedad al cual se secan las chapas, antes del proceso de fabricación del tablero.

El tamaño de los defectos inherentes a la madera se reduce tomando el espesor de la chapa y, además se dispersan. Como consecuencia de ello son menos influyentes sobre la resistencia.

Debido a que dos chapas adyacentes tienen normalmente sus fibras perpendiculares entre sí, los tableros de contrachapado poseen resistencias bastante parecidas en la dirección longitudinal y transversal, en comparación con la madera. Obviamente, esto también significa que el contrachapado es menos resistente que una pieza de madera solicitada en dirección paralela a la fibra. Las otras ventajas del contrachapado son: gran resistencia al agrietamiento y a la aparición de rajaduras, alta resistenca al cizalle a través del espesor y gran estabilidad dimensional.

\section{PROPIEDADES ESTRUCTURALES DEL CONTRACHAPADO}

La naturaleza de una franja de contrachapado es tal que la resistencia y rigidez de las chapas que tienen sus fibras en dirección normal a la dirección de la solicitación, contribuyen poco a la resistencia y rigidez del tablero.

Una buena aproximación a las reales propiedades estructurales se puede alcanzar tomando en cuenta sólo las chapas con fibras paralelas a la dirección de la solicitación. De esta forma, es posible calcular las propiedades estructurales del contrachapado una vez que se conozcan las propiedades de cada chapa. Con esta aproximación, el contrachapado se considera como una colección de piezas de madera, solicitadas por compresión paralela a la fibra. Resulta factible usar para el contrachapado la misma tensión admisible usada para la madera.

Cuando la solicitación está desangulada en $45^{\circ}$ respecto a la fibra de la chapa exterior y es necesario considerar las tensiones de cizalle, la estimación anterior no resulta recomendable. Es más conveniente basar el cálculo de esta tensión utilizando la sección transversal total, deduciendo las tensiones admisibles directamente de datos experimentales obtenidos de ensayos con contrachapado. 


\section{REQUERIMIENTOS GENERALES DEL CONTRACHAPADO}

La norma australiana AS 2269 establece calidades de chapas, calidad del adhesivo, uniones de chapas, tolerancia de las dimensiones y tensiones admisibles. Una breve revisión de esta norma permitirá aclarar los requisitos generales que, en países desarrollados, se le exigen al contrachapado estructural.

\section{Calidad de Chapas}

Las calidades de chapas que se establecen son cinco : Calidad A, Calidad S, Calidad B, Calidad C y Calidad D. Las características de ellas son:

- Calidad A. Describe una alta calidad del aspecto o apariencia de la chapa, apto para usarla sin revestimiento alguno por su característica de estar libre de defectos. Se recomienda en contrachapados donde el aspecto decorativo es importante, además de su comportamiento estructural y de su confiabilidad.

- Calidad S. Define una calidad de su aspecto que permite la presencia de características naturales de la madera, tales como nudos, que para el usuario son estéticas. Para ello se necesita el acuerdo entre ambas partes, productor y comprador, materializado en unas especificaciones escritas y aprobadas por los interesados, definiendo el tamaño y frecuencia aceptables de dichas características.

- Calidad B. Es una calidad por aspecto apto para dar, con pintura, una terminación de alta calidad, o dicho de otra, forma la superficie de la chapa debe ser capaz de aceptar pinturas y quedar con gran calidad de terminación.

- Calidad C. A esta calidad no se le exige en su apariencia o aspecto, pero debe tener una superficie sólida. Todos los defectos abiertos que existan en la chapa, antes de la aplicación del adhesivo, tales como agujeros y grietas, deben ser rellenados. Contrachapados con caras Calidad $\mathrm{C}$ se requieren para aplicaciones en donde se hace necesaria una superficie sólida. que no necesita ser decorativa. como es el caso del contrachapado que se pone en el piso para ser recubierto con alfombra, etc.

- Calidad D. A esta calidad no se le exige en su apariencia o aspecto, y se le permiten defectos abiertos tales como nudos, agujeros, grietas, etc., siempre que no sobrepasen 75 $\mathrm{mm}$ de ancho, medidos en dirección perpendicular a la fibra. Por apariencia, corresponde a la inferior calidad de las chapas. Se utiliza en aplicaciones en las cuales el aspecto no es prioritario pero si el comportamiento estructural, como es el caso de cerchas, vigas ocultas, pies-derechos en paneles de muro. arriostramientos, etc.

El contrachapado estructural debe ser clasificado de acuerdo a la calidad por apariencia de las superficies de las caras exteriores. 
Las superficies exteriores del contrachapado estructural pueden ser: calidad C y calidad D y deben corresponder, respectivamente, a chapas de calidad C y calidad D.

Las calidades obtenidas en la clasificación por aspecto del contrachapado estructural deben ser designadas por una combinación de letras que ser̃alan la calidad de la chapa de la cara (primera letra) y de la contracara (segunda letra), como sigue :

CC CD DD

Pueden especificarse, también, calidades por aspecto superiores. Estos tipos corresponden a chapas con calidad A, calidad S y calidad B, pero todos ellos deben clasificarse, estructuralmente, como Calidad D. Por ejemplo, si un tablero tiene Calidad $S$ en la cara y Calidad D en la contracara, el contrachapado estructural tendrá como clasificación final: DD.

Las especificaciones particulares de las calidades se presentan en el Cuadro $\mathbf{N}^{0} 1$.

\section{Tipo de Unión Encolada}

El tipo de unión encolada depende del adhesivo aplicado en las chapas. Según éste se tienen: Unión tipo A, unión tipo B, unión tipo C y unión tipo D. Las características de los distintos tipos son las siguientes:

- Unión tipo A. Se obtiene con un adhesivo basado en una resina sintética: fenolformaldehido, y fragua en forma definitiva bajo temperatura y presión controlada. La unión que origina con el contrachapado no se deteriora bajo condiciones húmedas, bajo calor o frío. Se le reconoce por su color café oscuro. Este tipo de unión se especifica para contrachapado marino, para contrachapado estructural y para contrachapado exterior, usado en condiciones de largas y severas exposiciones en ambientes húmedos (exteriores) o sumergido en agua.

- Unión tipo B. Obtenido con un adhesivo fabricado en base a la resina sintética melamina-formaldehido, fortificada con urea-formaldehido, y fragua también bajo temperatura y presión controlada. Esta unión se especifica para contrachapados que se someterán a condiciones húmedas de exteriores que involucran un período de hasta dos años de duración, tales como contrachapados para moldajes, o usados en aplicaciones sometidas a humedad intermitente, como es el caso de recubrimientos de puertas al exterior. 


\section{Cuadro $\mathrm{N}^{0} 1$}

\section{REQUISITOS DE LAS CHAPAS QUE CONFORMAN EL CONTRACHAPADO ESTRUCTURAL}

\begin{tabular}{|c|c|c|}
\hline \multirow[t]{2}{*}{ Defectos } & \multicolumn{2}{|c|}{ Calidad de la chapa } \\
\hline & $\mathrm{C}$ & D \\
\hline $\begin{array}{l}\text { Pudrición y atuque } \\
\text { activo de insectos }\end{array}$ & \multicolumn{2}{|c|}{ No se socptan } \\
\hline $\begin{array}{l}\text { Fallas en las uniones } \\
\text { de canto, grietas, } \\
\text { egujeros, perches, } \\
\text { bolsillos de cortezn y } \\
\text { de resines, éreas de } \\
\text { pudrición inactiva. }\end{array}$ & \multicolumn{2}{|c|}{ Las dimensiones acumuladas, en una longitud de $300 \mathrm{~mm}$, no deben exceder de $75 \mathrm{~mm}$. } \\
\hline Chepess & \multicolumn{2}{|c|}{$\begin{array}{l}\text { Deben cortarse suave y firmemente a un espesor constante. } \\
\text { Pueden ir en miss de una pieza y no necesitan ser del mismo color. }\end{array}$} \\
\hline $\begin{array}{l}\text { Bolsillos de corteza } \\
\text { goma y resina }\end{array}$ & \multirow{3}{*}{$\begin{array}{l}\text { Individualmente no deben exceder de un } \\
\text { ancho (medido en dirección normal a la fibra) } \\
\text { igual a } 6 \mathrm{~mm} \text { o de una superficie igual a } 2.000 \\
\mathrm{~mm}^{2}\end{array}$} & $\begin{array}{l}\text { Individualmente no deben exceder de un ancho } \\
\text { (modido en dirección normal a la fibra) igual a } 75 \mathrm{~mm} \\
\text { o de uns superficie igual a } 15.000 \mathrm{~mm}^{2}\end{array}$ \\
\hline $\begin{array}{l}\text { Uniones de canto sin } \\
\text { relleno }\end{array}$ & & $\begin{array}{l}\text { Individualmente no deben exceder de } 6 \mathrm{~mm} \text { (medido } \\
\text { en dirección normal a la fibra) o de uns superficie igual } \\
\text { a } 4.000 \mathrm{~mm}^{2}\end{array}$ \\
\hline Rajaduras sin relleno & & $\begin{array}{l}\text { Individualmente no deben exceder de } 9 \text { mm de ancho, } \\
\text { medidos en dirección normal a la fibra y de un largo } \\
\text { igual a la mitad de la longitud del tablero, medido en } \\
\text { dirección de la fibra } \\
\text { Individualmente no deben exceder de } 12 \mathrm{~mm} \text { de } \\
\text { ancho, medidos en dirección normal de la fibra y de un } \\
\text { largo igual a la } 1 / 3 \text { de la longitud del tablero, medido } \\
\text { en dirección de la fibra. }\end{array}$ \\
\hline $\begin{array}{l}\text { Uniones de canto y } \\
\text { rajacturas con relleno }\end{array}$ & $\begin{array}{l}\text { Individualmente no deben exceder de un } \\
\text { ancho (medido en dirección normal a la fibra) } \\
\text { igual a } 9 \mathrm{~mm} \text { o de una superficie igual a } 6.000 \\
\mathrm{~mm}^{2} \text {. }\end{array}$ & Se aceptan sin limitaciones. \\
\hline Agujeros sin relleno & $\begin{array}{l}\text { Individualmente no deben exceder de un } \\
\text { ancho de } 12 \mathrm{~mm} \text { (medido en dirección } \\
\text { normal a la fibra) y, considerando la suma } \\
\text { acumulada de sus superficies, ésta no debe } \\
\text { exceder de igual a } 6 \mathrm{~mm} \text { o de } 400 \mathrm{~mm}^{2} \text { por } \\
\text { tablero. }\end{array}$ & \multirow{2}{*}{$\begin{array}{l}\text { Individualmente no deben exceder de un ancho de } 75 \\
\text { mm (medido en dirección normal de la fibra) y } \\
\text { considerando la suma acumulada de sus superficies, no } \\
\text { debe exceder de } 15.000 \mathrm{~mm}^{2} \text { por tablero. }\end{array}$} \\
\hline Agujeros con relleno & $\begin{array}{l}\text { Individualmente no deben exceder de un } \\
\text { ancho de } 50 \mathrm{~mm} \text { (medido en dirección } \\
\text { normal a la fibra) y considerando la suma } \\
\text { acumulada de sus superficies, no deben } \\
\text { exceder de } 7.500 \mathrm{~mm}^{2} \text { por tablero. }\end{array}$ & \\
\hline Nudos & $\begin{array}{l}\text { Individualmente no deben exceder de } 50 \mathrm{~mm} \text {, } \\
\text { medidos en dirección normal a la fibra. }\end{array}$ & $\begin{array}{l}\text { Individualmente no deben exceder de } 75 \mathrm{~mm} \text {, medidos } \\
\text { en dirección normal a la fibra. }\end{array}$ \\
\hline Parches simples & $\begin{array}{l}\text { No deben exceder de } 75 \mathrm{~mm} \text {, medidos en } \\
\text { dirección normal a la fibra }\end{array}$ & $\begin{array}{l}\text { No deben exceder de } 100 \mathrm{~mm} \text {, medidos en dirección } \\
\text { normal a la fibra. }\end{array}$ \\
\hline $\begin{array}{l}\text { Manchas y } \\
\text { decoloración }\end{array}$ & \multicolumn{2}{|c|}{ Se aceptan sin limitaciones } \\
\hline $\begin{array}{l}\text { Escurrimiento de } \\
\text { adhesivo }\end{array}$ & \multicolumn{2}{|c|}{ Se aceptan sin limitaciones } \\
\hline $\begin{array}{l}\text { Desviación de la fibra } \\
\text { (sólo en contrachapado } \\
\text { clasificado } \\
\text { visualmente) }\end{array}$ & \multicolumn{2}{|c|}{$\begin{array}{l}\text { No debe exceder el tamaño } 1: 7 \text { si se extiende en más de } 1 / 4 \text { del área del tablero. } \\
\text { No se limita si se localiza en la vecindad de nudos y de otros defectos. }\end{array}$} \\
\hline Resina & \multicolumn{2}{|c|}{ Se aceptan sin limitaciones } \\
\hline $\begin{array}{l}\text { Rugosidad de la } \\
\text { superficie }\end{array}$ & \multicolumn{2}{|c|}{ Leve } \\
\hline
\end{tabular}


- Uniónes tipos C y D. Ambos tipos son para condiciones de uso en interiores y no se recomiendan para contrachapados que estarán en el exterior o sometidos a exposición de humedad o donde existan solicitaciones permanentes de aplastamiento. Aún cuando se usan en interiores los tipos de uniones C y D, no se recomiendan para contrachapado estructural. La línea de encolado tiene un leve coloreado. Estos tipos de uniones utilizan un adhesivo a base de urea-formaldehido que fragua bajo condiciones controladas de temperatura y presión. En resumen, estas uniones encoladas son aconsejables para contrachapados de uso interior no estructural.

Los contrachapados estructurales deben tener una union tipo A, usando un adhesivo a base de fenol-formaldehido.

\section{Uniones de Chapas}

- Uniones de Extremos. Cuando las chapas se unen por sus extremos, estas uniones deben ser achaflanadas, con una inclinación no mayor que 1:6, y el encolado de unión de extremos debe ser efectuado con un adhesivo de tipo fenólico.

- Uniones de los Cantos. Las uniones de los cantos de las chapas no necesitan ser encoladas, pero ellas deben cumplir con los requisitos establecidos para la calidad de las chapas.

- Uniones en los Tableros Contrachapados. Las uniones en los extremos y en los cantos de trozos de tableros por unir para conformar un sólo tablero, deben efectuarse con corte de pluma (chaflán), realizado a través de todo el espesor del tablero, de acuerdo a los siguientes requisitos:

Los tableros por unir deben ser fabricados con iguales materiales y proceso de fabricación.

Los chaflanes deben efectuarse a través de todo el espesor de ambos tableros.

La inclinación de los chaflanes no debe ser mayor que 1:10 para tableros de espesor menor que $12 \mathrm{~mm}$ y 1:8 para tableros de $12 \mathrm{~mm}$ o más de espesor.

Las chapas ubicadas en las caras de los tableros que se unen deben tener igual dirección.

El encolado de las uniones achaflanadas de los tableros que se unen debe efectuarse con un adhesivo tipo fenólico.

- Encolado entre Chapas. Como se dijo anteriormente, el encolado entre chapas debe ser del tipo A y debe cumplir con los siguientes requisitos:

El adhesivo usado en la fabricación del contrachapado estructural, debe ser del tipo fenólico.

El contrachapado entre chapas debe ser continuo sobre toda el área del tablero. 
- Ensamblado del contrachapado. La construcción deberá cumplir con los siguientes requisitos :

La construoción debe ser balanceada.

La dirección de las fibras, en chapas adyacentes, debe quedar formando un ángulo de aproximadamente $90^{\circ}$, con excepción del caso en que el número de chapas sea par. Las fibras de las chapas centrales deben quedar en la misma dirección.

Los tableros deben quedar libres de cualquier material metálico, tales como clips o corchetes.

- Espesor de las chapas en el tablero. El espesor nominal de las chapas debe ser de: 1,4 $\mathrm{mm} ; 1,6 \mathrm{~mm} ; 2,5 \mathrm{~mm}$ o $3,2 \mathrm{~mm}$. Se deben determinar las propiedades de las secciones transversales para estos espesores ${ }^{(2)}$.

- Sopladuras. Se permiten las sopladuras en las chapas centrales, resultantes del uso de la calidad D o de chapas de distinta calidad.

- Traslapos y Pliegues. Se admiten, siempre que la resistencia o utilidad del tablero no se reduzca. En los tableros no destinados a uso estructural, las chapas más compactas deben ubicarse en las caras exteriores.

\section{Dimensiones y Formas}

- Dimensiones. Las dimensiones reales de los tableros no deben diferir de las dimensiones solicitadas, en más de las siguientes tolerancias:

Espesor ${ }^{(3)}$. Para tableros no lijados con un espesor hasta $6 \mathrm{~mm}$, inclusive, más o menos $5 \%$. Para tableros no lijados con un espesor superior a $6 \mathrm{~mm}$ más o menos $3 \%$ y para tableros lijados una tolerancia adicional de $-0,3 \mathrm{~mm}$ por cara lijada.

Longitud y Ancho, más o menos $1.5 \mathrm{~mm}$.

- Forma. Cuando se procede a medir un tablero contrachapado, éste debe cumplir con los siguientes requisitos:

Cuadratura o Rectangularidad. La diferencia entre longitudes de las dos diagonales del tablero no debe exceder de $0,2 \%$ de la longitud de la diagonal más larga.

(2) Pueden ser usados otros espesores de chapas, siempre que los detalles de éstos y el tipo de construcción sean informados por el fabricante, de tal modo que permitan calcular las propiedades geométricas de las secciones transversales.

(3) Las dimensiones del espesor se aplican a tableros sin recubrimientos u otra capa sobre el tablero, como barniz. pintura, papel. etc. 
Rectitud de los Bordes. Cualquier borde del tablero no debe desviarse de la línea recta en más del $0,05 \%$ de la longitud de dicho borde.

Lisura. Para un tablero no cargado, la distancia máxima entre cualquier punto del tablero y un plano horizontal no debe exceder de $50 \mathrm{~mm}$. Cuando el tablero se carga y tiene hasta $12 \mathrm{~mm}$ de espesor, $10 \mathrm{Kg}$. Para tableros que exceden los 12 mm de espesor, $15 \mathrm{Kg}$. El tablero debe tocar la superficie horizontal justo debajo (según una vertical) del área cargada.

Terminaciones. A menos que se especifique de otra forma, cada tablero contrachapado estructural debe tener su cara y trascara no pulida o lijada, y libre de parches. Los bordes deben estar cortados limpiamente y perpendiculares a las caras. Son permitidos los defectos de aserrado ubicados hasta $5 \mathrm{~mm}$ del borde.

Uniones en los Tableros. Las uniones de trozos de tableros, para conformar un contrachapado estructural, puden ser convenidas entre comprador y vendedor.

\section{Contenido de Humedad}

En el momento del despacho del contrachapado, el contenido de humedad, en cualquier punto del tablero, debe ser:

- Para tableros que no excedan de $7 \mathrm{~mm}$ de espesor, no menos que $10 \%$ y no más que $15 \%$

- Para tableros que excedan de $7 \mathrm{~mm}$ de espesor, no menos que $8 \%$ y no más que $15 \%$

- En caso de controversia, el contenido de humedad debe determinarse por el método de secado en estufa, especificado en la norma correspondiente.

\section{CLASIFICACIÓN ESTRUCTURAL DE LOS TABLEROS}

Los tableros de contrachapado estructural, fabricados con chapas de calidad C y calidad $\mathrm{D}$, deberán ser clasificados por uno de los dos procedimientos descritos a continuación :

\section{Método de la Densidad de las Chapas}

Para tableros de contrachapado estructural construidos de chapas provenientes de una especie maderera para la cual no se ha definido una clase estructural, se debe determinar la densidad media de las chapas provenientes de esa especie y con la ayuda del Cuadro $\mathrm{N}^{\circ} 2$, encontrar la clase estructural que le corresponde a dicha madera.

Los valores de las Tensiones Admisibles, que corresponden a cada clase estructural, son los incluidos en la Norma NCh 1198, Cuadros $\mathrm{N}^{\circ 8} 3 \mathrm{~A}$ y $3 \mathrm{~B}$. 


\section{Cuadro $\mathrm{N}^{\circ} 2$.}

\section{CLASE ESTRUCTURAL DETERMINADA EN BASE A LA DENSIDAD DE LA CHAPA EXTRAIDA DE LA MADERA USADA.}

\begin{tabular}{|c|c|}
\hline $\begin{array}{c}\text { Densidad Aparente } \\
\left(\mathrm{Kg} / \mathrm{m}^{3}\right)\end{array}$ & Clase estructural \\
\hline 1.200 & $\mathrm{~F} \mathrm{34}$ \\
\hline 1.080 & $\mathrm{~F} \mathrm{27}$ \\
\hline 960 & $\mathrm{~F} \mathrm{22}$ \\
\hline 840 & $\mathrm{~F} 17$ \\
\hline 730 & $\mathrm{~F} 14$ \\
\hline 620 & $\mathrm{~F} 11$ \\
\hline 520 & F 8 \\
\hline 420 & F 7 \\
\hline
\end{tabular}

- Valores promedios obtenidos de determinaciones de densidad en a lo menos 5 trozas de una especie determinada o, cuando no están disponibles 5 trozas. el valor promedio se obtiene de a lo menos 5 chapas escogidas al azar, del total de chapas cortadas de una troza o de trozas de una especie determinada.

Cuadro $\mathrm{N}^{\circ} 3 \mathrm{~A}$

TENSIONES ADMISIBLES DE LAS CLASES ESTRUCTURALES.

\begin{tabular}{|c|c|c|c|c|c|c|c|}
\hline \multirow{3}{*}{$\begin{array}{c}\text { Clase } \\
\text { Estructural }\end{array}$} & \multicolumn{7}{|c|}{ Tensión Admisible (MPa) } \\
\hline & \multirow[b]{2}{*}{ Flexión } & \multirow[b]{2}{*}{ Tracción } & \multirow[b]{2}{*}{ Cizalle } & \multicolumn{2}{|c|}{ Compresión } & \multirow[b]{2}{*}{ Ef } & \multirow[b]{2}{*}{ G } \\
\hline & & & & $\begin{array}{c}\text { Fn el plano del } \\
\text { tahlero }\end{array}$ & $\begin{array}{l}\text { Perpendicular al } \\
\text { plano del tablero }\end{array}$ & & \\
\hline F 34 & 34.5 & 27.5 & 2.3 & 25.9 & 10.4 & 18.150 & 908 \\
\hline F 27 & 27.5 & 22.0 & 2.3 & 20.6 & 9.0 & 15.000 & 750 \\
\hline F 22 & 22.0 & 17.0 & 2.3 & 16.5 & 7.8 & 12.600 & 630 \\
\hline F 17 & 17,0 & 14.0 & 2.3 & 12.8 & 6.6 & 10.600 & 530 \\
\hline F 14 & 14,0 & 11.0 & 2.1 & 10.5 & 5,2 & 9.100 & 455 \\
\hline F 11 & 11.0 & 8.6 & 1.8 & 8.3 & 4.1 & 7.900 & 395 \\
\hline F 8 & 8.6 & 6.9 & 1.6 & 6.5 & 3.5 & 6.900 & 345 \\
\hline F 7 & 6,9 & 5.5 & 1.4 & 5.2 & 2.6 & 6.100 & 305 \\
\hline
\end{tabular}




\section{Método de Clasificación Estructural Mecánica}

Es un método de clasificación no destructivo que permite establecer la clase estructural de un tablero contrachapado, determinando la relación carga-deformación.

Cuadro $\mathrm{N}^{\circ}$ 3B.

TENSIONES ADMISIBLES DE LOS GRADOS ESTRUCTURALES DEL PINO RADIATA.

\begin{tabular}{|c|c|c|c|c|c|c|c|}
\hline \multirow{3}{*}{$\begin{array}{c}\text { Clase } \\
\text { Estructural }\end{array}$} & \multicolumn{7}{|c|}{ Tensión Admisible (MPa) } \\
\hline & \multirow[b]{2}{*}{ Flexión } & \multirow[b]{2}{*}{ Tracción } & \multirow[b]{2}{*}{ Cizalle } & \multicolumn{2}{|c|}{ Compresion } & \multirow[b]{2}{*}{ Ef } & \multirow[b]{2}{*}{$\mathbf{G}$} \\
\hline & & & & $\begin{array}{c}\text { En el plano del } \\
\text { tablero }\end{array}$ & $\begin{array}{l}\text { Perpendicular al } \\
\text { plano del tablero }\end{array}$ & & \\
\hline GS & 11,0 & 6,6 & 0,9 & 8,3 & 2.5 & 10.500 & 525 \\
\hline G1 & 7,5 & 4,5 & 0.7 & 5,6 & 2,5 & 9.000 & 450 \\
\hline G2 & 4.0 & 2.0 & 0,4 & 4,0 & 2,5 & 7.000 & 350 \\
\hline
\end{tabular}

El procedimiento es el siguiente:

- Determinar el espesor y ancho de la probeta (tablero).

- Determinar la luz (L), considerando que la razón minima entre luz/espesor debe ser 48.

- Determinar el Momento de Inercia (I) de todas las chapas que componen el tablero, considerando el ángulo que forma la dirección de la fibra, en la chapa, con la dirección de la solicitación.

- Colocar el tablero sobre los apoyos de modo que la dirección de la fibra de las caras exteriores sea paralela a la luz.

- Eliminar el posible error del cero. colocando una carga inicial en el centro de la luz.

- Aplicar una segunda carga conocida $(\mathrm{P})$ y leer la deformación $(\Delta)$ que corresponda a dicha carga.

- Calcular el módulo de elasticidad ( Ef ) del tablero, usando la expresión:

$$
E f=\frac{P^{*} L^{3}}{48^{*} \Delta^{*} I}
$$

- Con el módulo de elasticidad así calculado. determinar, en los Cuadros N³A o N³B la clase o calidad estructural del tablero en estudio. 


\section{IDENTIFICACIÓN DEL TABLERO CONTRACHAPADO}

Cada tablero de contrachapado estructural debe contener la siguiente información, en forma legible $\mathrm{e}$ indeleble, por lo menos una vez.

a) Nombre del fabricante o su marca registrada.

b) La palabra "Estructural" o la descripción del producto.

c) La calidad del aspecto de las chapas exteriores (la cara primero y luego la contracara: $\mathrm{CC}, \mathrm{CD}$ o DD.

d) El tipo de encolado aplicado entre chapas, por ejemplo: "Unión Tipo A".

e) Una indicación de la calidad estructural del tablero.

Ejemplo : "F T" o una marca con el color apropiado. Cada una de ellas puede correlacionarse con un color. La Norma Australiana establece:

$\begin{array}{llll}\text { F 7 } & \text { : Azul } & \text { F 17 } & \text { : Amarillo } \\ \text { F 8 } & \text { : Verde } & \text { F 22 } & \text { : Blanco } \\ \text { F 11 } & \text { : Pirpura } & \text { F 27 } & \text { : Sin color } \\ \text { F 14 } & \text { : Naranja } & \text { F 34 } & \text { : Sin color }\end{array}$

f) Si el tablero recibe un tratamiento preservador, éste debe cumplir con la correspondiente norma chilena sobre preservación.

g) Los requisitos: b), c), d) y e) deben colocarse en la siguiente forma típica:

$$
\begin{gathered}
\text { ESTRUCTURAL } \\
\text { CD - UNION A } \\
\text { F } 7
\end{gathered}
$$

Ésto indica: Contrachapado estructural, con cara calidad C, trascara calidad D, unión encolada Tipo A y calidad estructural $\mathrm{F} 7$

Se advierte que la presencia de la identificación señalada en el contrachapado, en el empaquetado o en la literatura relacionada con la adquisición puede tomarse como una afirmación del fabricante que su producto asi marcado cumple todas las especificaciones normalizadas. Es conveniente que el comprador se proteja con un Certificado de Control de Calidad obtenido mediante un muestreo y ensayos de la muestra extraida. En tal caso el Informe debe contener, a lo menos, lo siguiente: Dimensiones de los tableros que constituyen la muestra, número de tableros controlados. Contenido de Humedad de los tableros muestreados, luz de ensayo en mm, carga aplicada. deformación obtenida con tal carga y referencia a la norma con cuyas especificaciones se realizó el control. 


\section{PROPIEDADES GEOMÉTRICAS DEL CONTRACHAPADO}

Debido a que el contrachapado está constituido, generalmente,por un número impar de láminas superpuestas, con diferentes direcciones de fibra, usualmente ortogonales, se originan propiedades mecánicas distintas dependientes de la orientación de la fibra en las chapas externas y, más aún, cuando las láminas que conforman un mismo tablero provienen de especies diferentes. Por otra parte, aunque un tablero esté fabricado con chapas de la misma especie. la calidad de las interiores resulta de inferior calidad que aquellas ubicadas en las caras exteriores.

Para enfrentar el estudio de un material compuesto por láminas con propiedades mecánicas diferentes. se ha recurrido a tres métodos:

"Método de la Sección Transversal Efectiva" o de las capas paralelas.

"Mćtodo de la Sección Transversal Transformada". y

"Método de la Sección Transversal Bruta".

\section{Método de la Sección Transversal Efectiva}

Desarrollado en Estados Unidos. por Freas y Liska. ha sido usado durante mucho tiempo en ese pais y Canadá. Este método sólo considera como secciones efectivas de una sección transversal a las chapas cuyas fibras tienen la misma dirección de los esfuerzos principales.

Los ensayos realizados, para avalar esta teoria, demuestran que en muchos casos prácticos la aproximación es aceptablemente buena. Las mejores predicciones se obtienen en compresión y en tracción, y las más deficientes. en flexión. La peor aproximación resulta para el caso en que la fibra de la chapa cxterna se dispone en dirección perpendicular a la luz. resultando un momento resistente un $50 \%$ mayor que el previsto en forma teórica.

Entre las desventajas de éste método se pueden mencionar : La no aplicabilidad para casos donde los esfuerzos principales queden en direcciones diferentes de $0^{\circ}$ ó de $90^{\circ}$. con respecto a la chapa externa. y cuando el contrachapado es solicitado al corte.

\section{Método de la Sección Transversal Transformada}

Este método es similar al anterior. pero se diferencia de él al considerar el aporte de las chapas cuyas fibras son perpendiculares a la dirección de los esfuerzos principales. Tiene la ventaja sobre el anterior. que permite describir el comportamiento para cualquier ángulo entre la dirección de la fibra de la chapa exterior (f.c.e.) y la dirección de los esfucrzos principales. 
Este procedimiento considera, para las chapas cuya dirección de la fibra es diferente al esfuerzo principal, una fracción de la propiedad mecánica aplicable a la chapa si ésta tuviese la dirección de sus fibras paralela al esfuerzo principal. Las propiedades mecánicas para las cuales opera este método, son :

Módulo de elasticidad para compresión, tracción y flexión; y Módulo de Corte, G, para el cizalle.

Para determinar las propiedades geométricas, se considera un ancho efectivo menor en aquellas láminas que no tienen la misma propiedad mecánica de las chapas externas. Estas diferencias ocurren cuando las láminas tienen una dirección de fibra diferente al esfuerzo principal. o bien en chapas internas de diferente especie maderera o de distinta calidad.

El ancho se reduce en función de la propiedad mecánica de las chapas externas, si éstas tienen sus fibras en la dirección del esfuerzo principal.

Para la aplicación de los dos métodos ya analizados, se debe evaluar, para cada espesor de tablero, las propiedades geométricas mínimas por ser usadas en la verificación o diseño compatibles con la norma de fabricación.

\section{Método de la Sección Transversal Bruta}

Fue propuesto en 1963 por el investigador sueco E. Niskanen, como alternativa a los métodos anteriores, con el objeto de obviar la complejidad inherente a su uso práctico.

En el método de la sección transversal bruta las tensiones básicas se determinan de ensayos realizados en probetas pequeñas extraidas de tableros comerciales.

Entre las ventajas de éste método, la más importante es que no tiene cabida la subestimación de las propiedades geométricas del material, como ocurre con el " Método de la Sección Transversal Efectiva". Por otra parte. al considerar la sección transversal neta. las propiedades mecánicas no están sujetas directamente a las dimensiones de la chapa, como ocurre con los métodos anteriormente presentados.

Tanto las relaciones que entrega la American Plywood Asociation (A.P.A.), como las fórmulas empiricas norteamericanas no garantizan una aplicabilidad a los tableros fabricados con especies madereras crecidas en Chile. Por lo tanto, se hace necesario conocer el comportamiento del contrachapado nacional, utilizando los tres métodos ya señalados.

Para obtener información con propósitos de diseño de elementos estructurales, las probetas de ensayo deberán tener un número suficiente de caracteristicas reductoras (defectos). localizadas apropiadamente. Pero resulta difícil establecer reglas generales para el muestreo o extracción de probetas de cada tablero. Sólo es posible controlar los defectos de las chapas externas. Se desconoce la correlación entre los resultados de 
probetas pequeñas, donde se incluya defectos permitidos, y el comportamiento de los componentes estructurales de tamaño mayor. Por lo expuesto anteriormente, lo normal es ensayar probetas libres de defectos en sus caras exteriores.

\section{REFERENCIAS.}

Instituto Forestal, 1981. Propiedades Mecánicas y Asociadas del Contrachapado Fabricado en Chile. Informe Técnico $\mathrm{N}^{\circ} 81$, Santiago, Chile.

De Urruticoechea S., Carlos, 1984. Propiedades Mecánicas de un Contrachapado Estructural de Tepa y Olivillo. Memoria para optar al titulo de Ingeniero Civil, Facultad de Ciencias Físicas y Matemáticas, Universidad de Chile, Santiago.

Alegria M. y González S., 1990. Estudio de las Propiedades Mecánicas del Contrachapado Estructural INFODEMA S.A. Memoria para optar al titulo de Ingeniero Civil en Obras Civiles, Facultad de Ingenieria, Universidad de Santiago de Chile, Santiago.

Boding J.and Joyne B., 1982. Mechanics of Wood and Wood Composites. Published by Von Nostrand Reinold Company Inc., New York. U.S.A.

American Society for Testing and Materials, 1992. ASTM D-4442, Standard Test Methods for Direct Moisture Content Measurement of Wood and Wood

American Society for Testing and Materials, 1987. ASTM D-2395, Standard Test Methods for Specific Gravity of Wood and Wood-base Materials.

American Society for Testing and Materials, 1987. ASTM D-3501, Standard Methods of Testing Plywood in Compression.

American Society for Testing and Materials, 1987. ASTM D-3500, Standard Methods for Structural Panels in Tension.

American Society for Testing and Materials, 1987. ASTM D-3043, Standard Methods of Testing Structural Panels in Flexure.

American Society for Testing and Materials, 1990. ASTM D-2718, Standard Test Method for Structural Panels in Planar Shear (Rolling Shear).

American Society for Testing and Materials, 1986. ASTM D-3044, Standard Test Method for Shear Modulus of Plywood

Standards Association of Australia, 1979. AS 2269, Structural Plywood.

Standards Association of Australia, 1977. AS 2097, Methods for Sampling Veneer and Plywood.

Standards Association of Australia, 1977. AS 2098, Methods of Test for Veneer and Plywood.

Standards Association of Australia, 1979. AS 2289, Glossary of Terms Used in the Plywood Industry.

Plywood Association of Australia Ltd. (PAA), 1985, Structural Plywood, Design Manual. 
Plywood Association of Australia Ltd. (PAA), 1994, New Joint Australian \& New Zealand Standard for Structural Plywood.

Plywood Association of Australian Ltd.,1992, T\&G Structural Plywood for Residential Flooring. Design Manual.

Standards Association of New Zealand, 1981, NZS 3615 Specification for Strength and Design Methods for Construction Plywood. Wellington, New Zealand.

Instituto Nacional de Normalizacion (INN), 1990, NCh 1198, Madera - Construcción en Madera - Cálculo. Santiago, Chile.

Haygreen J.G., and Bowyer J.L., 1989, Forest Products and Wood Science. An Introduction, Second Edition. Published lowa State University Press. 